\title{
Duodenal mucosal architecture in non-specific and ulcer-associated duodenitis
}

\author{
M H ASAN, * W SIRCUS. AND A N NE FERGUSON \\ From the Gastro-Intestinal Unit, Western General Hospital and University of Edinburgh, Edinburgh
}

SUMmary This study was done to determine the severity and extent of abnormalities of duodenal mucosal architecture in non-specific (non-ulcerative) and ulcer-associated duodenitis. The effect of successful treatment with cimetidine on these changes has also been assessed. A method of microdissection and measurement of villus height, crypt depth, and mitotic figure count per crypt was applied to endoscopic biopsies from the duodenum. Five groups of patients were studied: untreated ulcer-associated duodenitis, untreated non-specific duodenitis, healed ulcer-associated and non-specific duodenitis after cimetidine treatment, and controls. Significant reduction in villus height, increase in crypt length, and increase in mitotic figure count per crypt were all found in both ulcer-associated and severe non-specific duodenitis as compared with controls. These changes were localised to visually inflamed areas and regressed after healing of these lesions with cimetidine. This is the first quantitative comparison of the architectural features between diseased states in the duodenum and controls in the same study. Identical morphological changes in the form of crypt hyperplasia and villus atrophy were demonstrated in areas of non-specific and ulcer-associated duodenitis. No evidence could be found from this study that non-specific duodenitis constitutes a different disease from ulcerassociated duodenitis.

Visual and histological changes of inflammation often occur in localised areas of the proximal duodenum in association with peptic ulcer. Similar changes have been seen in the proximal duodenum in the absence of peptic ulceration and termed 'non-specific duodenitis'.' However, there is controversy as to whether non-specific duodenitis is an entity in its own right, or is always part of the spectrum of duodenal ulcer disease.

Subjective histopathological examination of endoscopic biopsies from patients with non-specific duodenitis has shown abnormalities in tissue morphology. Shortening of villi has been reported by several authors." " Crypt dilatation has been found by Gelzayd and co-workers." and other workers have reported reduction in crypt depth. ${ }^{2}$ Controversy also exists as to what constitutes 'normal' duodenal morphology"i and, in view of the rela-

*Address for correspondence and for requests for reprints: Dr Mahmud Hasan, Gastro-Intestinal Unit, Western General Hospital, Crewe Road, Edinburgh EH4 2XU. tively minor changes reported in these duodenal biopsies, objective measurements of the components of the duodenal mucosa (morphometry) should be helpful. However, in general, endoscopic biopsies which have been processed by conventional histological techniques are. because of the small size of the biopsies, trauma, and difficulty in proper orientation, unsuitable for morphometric examination.

We have recently described a technique which is suitable for morphometric study of the architecture of villi and crypts of endoscopic duodenal biopsies. ${ }^{7}$ The tissue is stained in bulk, villi and crypts dissected with the aid of a dissecting microscope, and their sizes and mitotic counts measured directly. This method has now been used to study: 1. the severity of mucosal architecture abnormalities in duodenitis, als defined on the basis of visual appearances at endoscopy;

2. differences between non-specific duodenitis and ulcer-associated duodenitis in terms of mucosal architecture;

3. the extent of abnormality of mucosal archi- 
tecture-that is, whether the abnormal villus architecture is localised to the visually abnormal part of the duodenum, or whether it involves the entire duodenum;

4. the effect of treatment with cimetidine on the mucosal abnormality.

\section{Methods}

P A T I E N T S

All patients had symptoms which were thought to be related to disease of the upper gastrointestinal tract, and endoscopic examination of the oesophagus, stomach and duodenum was carried out for diagnostic purposes. Duodenal biopsies from five groups of patients were studied: ulcerassociated duodenitis, non-specific duodenitis, healed ulcer-associated duodenitis after cimetidine treatment, healed non-specific duodenitis after cimetidine treatment, and controls.

All those in whom non-specific duodenitis was found had had symptoms which suggested to the clinician at an outpatient consultation that a duodenal ulcer was likely to be present. There were 20 patients in this group. Fifteen patients constituted the ulcer-associated duodenitis group. The healed ulcer-associated duodenitis after cimetidine treatment and healed non-specific duodenitis after cimetidine treatment patients had been treated with the conventional dose of cimetidine $(1 \mathrm{~g} /$ day $)$ for non-specific and ulcer-associated duodenitis disclosed at a previous endoscopy. The period of treatment varied from six to 12 weeks, and all were symptom-free at the time of the second endoscopy. There were 10 patients each in these two groups. These were not the same patients who formed the ulcer-associated duodenitis and nonspecific duodenitis groups. The control group consisted of 15 patients in whom the indication for endoscopy was the presence of symptoms to suggest oesophageal reflux, together with hiatus hernia, but who were found to have no endoscopic abnormality in the upper gastrointestinal tract apart from the hiatus hernia.

E N D O S C O P Y A N D B I O P S Y

Upper gastrointestinal endoscopy was carried out in the morning after overnight fasting in the usual way. The Olympus GIF-K oblique viewing endoscope was chosen because it permits the taking of biopsies of adequate size. The examination was done by any one of us and a grading system was used to classify duodenitis. Mild duodenitis was diagnosed when the mucosa of the duodenal bulb was mildly hyperaemic, swollen but without gross narrowing of the lumen, and with only slight contact bleeding. Severe duodenitis was diagnosed when the mucosa was intensely congested, the lumen was narrowed, and spontaneous petechial bleeding and multiple erosions were present. When

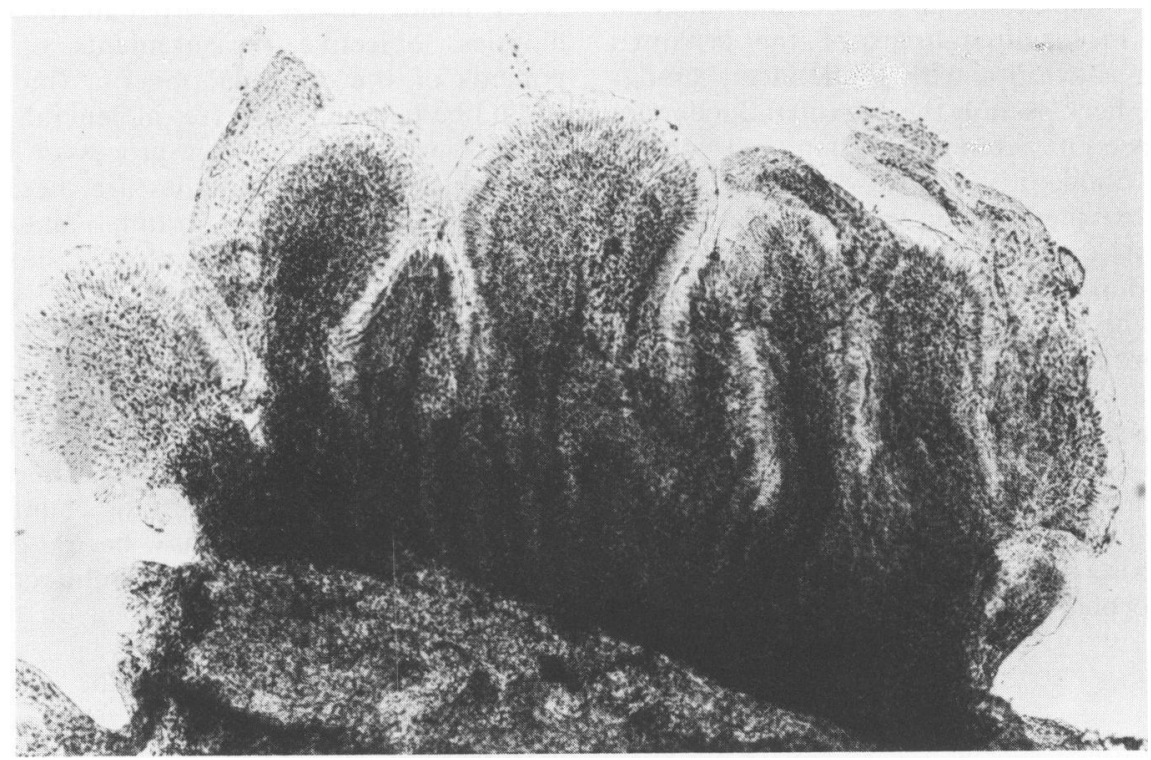

Fig. 1 Dissected strip of duodenal mucosa showing several villi and crypts $(S c h i f f \times 50)$. 
associated with one or more ulcer craters with visible depth and sharp borders similar changes were regarded as those of ulcer-associated duodenitis whether they were generalised or patchy.

Two or more per-endoscopic biopsies were taken from the following sites:

1. Control subjects-from the bulb and the distal second part of duodenum;

2. Ulcer-associated duodenitis patients-from areas of duodenitis away from the edge of the ulcer and from a visually unaffected part of the duodenal bulb. In 10 cases biopsies from the distal second part of duodenum were also taken;

3. Non-specific duodenitis patients-from visually affected and normal-appearing bulb and in 10 cases from the distal second part of duodenum;

4. Healed ulcer-associated duodenitis after cimetidine treatment and healed non-specific duodenitis after cimetidine treatment-from the bulb.

PRE PAR A T I O N OF SPECI MENSAND M I C R O D I S S E T I O N

Specimens were fixed in Clarke's fixative, then passed through $50 \%$ ethyl alcohol in water $(10$ minutes), tap water (10 minutes), molar hydrochloric acid at $60^{\circ} \mathrm{C}$ in a water bath (six minutes), three changes of tap water at room temperature (10 minutes), then Schiff's reagent at room temperature ( 20 minutes). Thereafter specimens were rinsed in water and examined within six hours.
Specimens were examined in tap water under the dissecting microscope. Strips of mucosa 2-3 $\mathrm{mm}$ long and one villus thick were cut free with the help of a cataract knife. Several such strips from each biopsy were placed on a slide in a drop of $45 \%$ acetic acid and covered with a coverslip (Fig. 1). The height of villi and the depth of crypts were then measured under a light microscope using an eyepiece graticule. Measurements of length of 10 villi and 10 crypts were made in each specimen and the means calculated. The preparation was then gently squashed to release the crypts from lamina propria and the number of mitotic figures in each of 10 crypts was counted (Fig. 2). All stages of mitosis were included in the mitotic count.

\section{Results}

Satisfactory preparations of duodenal biopsies were obtained in every case. Examination of two biopsies from each site was of ten needed to give 10 villi and crypts suitable for measurements. Villi of various shapes, finger, leaf, ridged, fused, were seen in biopsies from both normal and abnormal areas. It was not possible to distinguish normal and inflamed mucosa on the basis of the appearance of villi.

Significant differences emerged between the controls and ulcer-associated duodenitis in villus

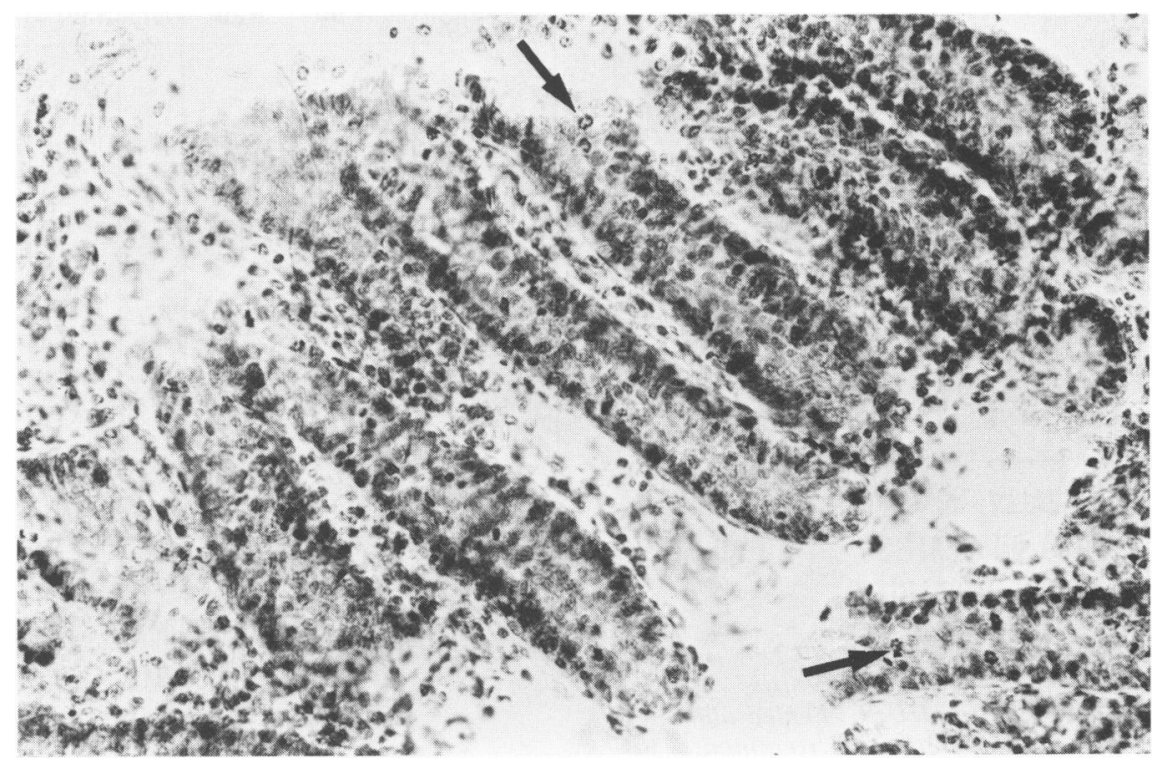

Fig. 2 Several crypts of Lieberkïhn separated from lamina propria of duodenal mucosa showing mitotic figures $($ Schiff $\times 160)$. 
height $(\mathrm{P}<0.001)$, this being shorter in the latter group (Fig. 3). Ulcer-associated duodenitis biopsies also showed increased depth of crypts $(P<0.001)$ and increased number of mitotic figures per crypt $(\mathrm{P}<0.01)$ as compared with the controls (Figs. 4, 5).

Comparison between the measurements obtained in severe non-specific duodenitis and controls also showed similar differences. Villi were shorter $(\mathrm{P}<0.01)$, crypt depth increased $(\mathrm{P}<0.001)$, and mitotic figures per crypt increased $(P<0.01)$ in severe non-specific duodenitis (Figs. 3, 4, 5).

Values between controls and mild non-specific duodenitis showed no significant difference in any of these parameters. In biopsies from visually uninvolved bulb mucosa in both ulcer-associated duodenitis and non-specific duodenitis, there were no significant differences in these measurements when compared with controls. Measurements in the biopsies from the second part of the duodenum showed no significant difference between controls and ulcer-associated duodenitis and non-specific duodenitis.

In cases where ulcer-associated and non-specific

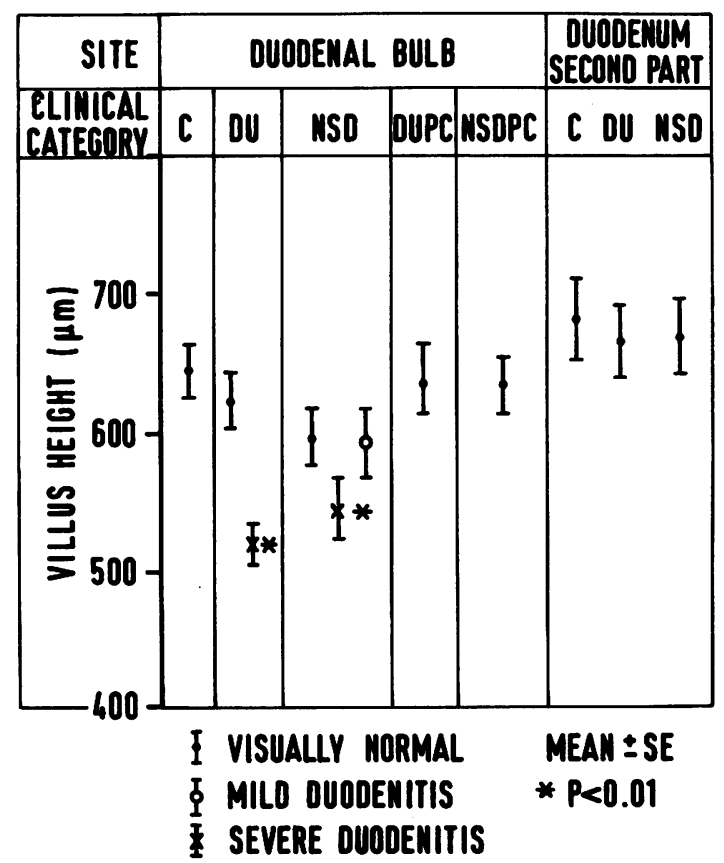

Fig. 3 Measurements of villus height in $\mu \mathrm{m}$. $C$ : controls, DU: ulcer-associated duodenitis, NSD: non-specific duodenitis, DUPC: healed ulcerassociated duodenitis after cimetidine treatment, NSDPC: healed non-specific duodenitis after cimetidine treatment.

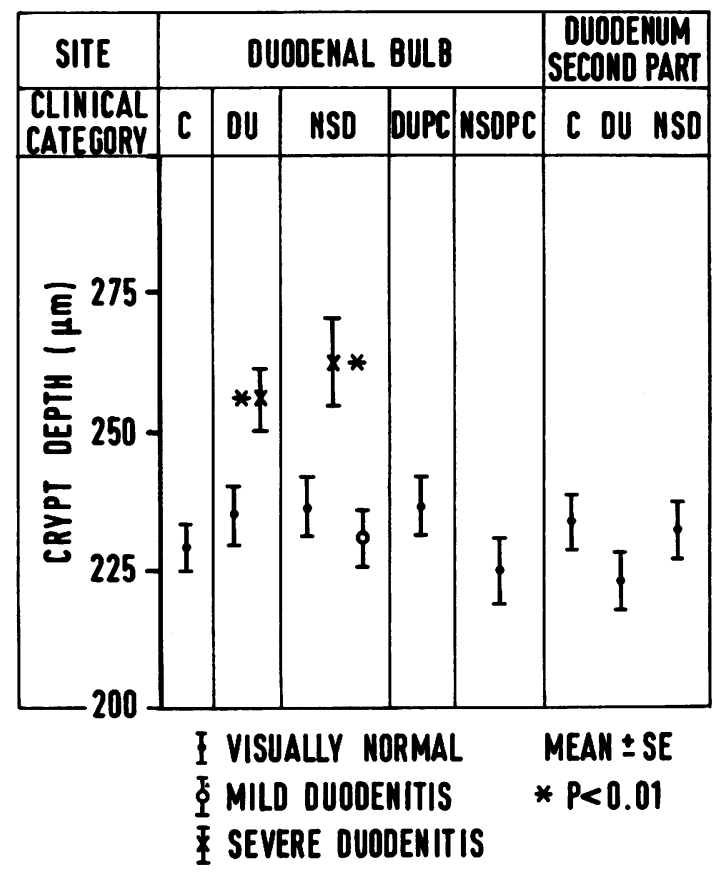

Fig. 4 Measurements of crypt depth in $\mu \mathrm{m}$. Abbreviations as in Fig. 3.

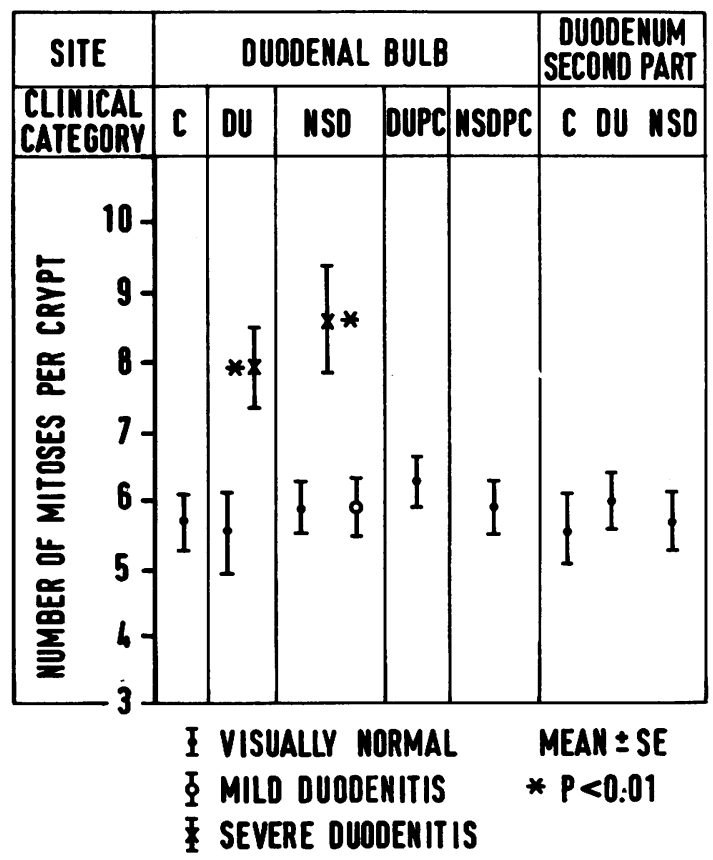

Fig. 5 Number of mitoses per crypt. Abbreviations as in Fig. 3. 
duodenitis had healed differences in these measurements compared with controls were no longer significant.

\section{Discussion}

The microdissection technique for measurement of intestinal crypt and villus length was found to be satisfactory for the study of perendoscopic duodenal biopsies. The results of measurements of the control specimens in this study are very similar to those described previously. ${ }^{7}$ Consistent abnormality in the mucosal architecture in the form of shortening of villi, lengthening of crypts, and increased number of mitoses in crypts have been demonstrated in visually defined non-specific as well as ulcer-associated duodenitis as compared with controls. These changes were identical in non-specific and ulcer-associated duodenitis. It is important to emphasise that architectural changes were not present throughout both the first and second parts of the duodenum, but, in fact, were found only in visually abnormal areas. The duodenal mucosal architecture returns to normal after successful treatment with cimetidine.

The objective measurements made in biopsies taken from areas of 'mild duodenitis' show that with regard to crypt and villus length and number of mitotic figures per crypt there are no significant differences from normal. Preliminary results in a parallel study of the cellular infiltrate in duodenitis also show no differences between mild duodenitis and normal. Although we cannot exclude the possibility that mild duodenitis represents an early stage in the evolution of the pathology of more severe duodenitis and duodenal ulceration, it seems more likely that the visually recognisable mild abnormalities seen at endoscopy mostly reflect changes in blood flow.

With respect to the previous conflicting evidence of crypt changes in non-specific duodenitis, ${ }^{245}$ we have in this study demonstrated that crypt length is increased, and that there is also an increase in the number of mitotic figures, both in non-specific and in ulcer-associated duodenitis. In the only previous reported attempt to measure cell proliferation directly, the authors found diminished ${ }^{3} \mathrm{H}-\mathrm{TdR}$ incorporation in vitro in biopsies obtained from five duodenal ulcer patients but the comparison control group contained only two subjects. It is also possible that proliferative activity of crypts at the edge of an ulcer crater may be different from that of crypts in adjacent areas of duodenitis.

Many factors can alter the small intestinal mucosal architecture of the small intestine." 10
Villus shortening and crypt hyperplasia may be caused by one of several mechanisms. On the one hand, primary damage to the villus cells may lead to compensatory hyperplasia in the crypts; alternatively, hormonal and/or immunological factors locally released can exert a direct effect on crypt cells, resulting in proliferation. The objective of this work was to document the changes in mucosal architecture and the study does not allow conclusions to be made about the mechanism of crypt hyperplasia or villus shortening. However, our finding that these changes are localised to visually abnormal parts of the duodenal bulb and return to normal after treatment with cimetidine points to a primarily luminal event as the cause of villus cell damage. Immunological, hormonal, and other factors may additionally contribute to tissue changes later in the evolution of duodenitis and a study on the nature of the cellular infiltrate in the duodenal mucosa is under way.

We are grateful to all the staff of the Endoscopy Theatre of the Western General Hospital, Edinburgh, for their help with the collection of the specimens. We also acknowledge the help of the Association of Commonwealth Universities who are financing the study of one of us (MH).

\section{References}

${ }^{1}$ Classen M, Koch H, Demling L. Duodenitis. Significance and frequency. Bibl Gastroenterol 1970; 9: 48-69.

${ }^{2}$ Cheli R. Duodenitis and duodenal ulcer. A biopsy study. Digestion 1968; 1:175-82.

${ }^{3}$ Whitehead R, Roca M, Meikle DD, Skinner J, Truelove SC. The histological classification of duodenitis in fibreoptic biopsy specimens. Digestion 1975; 13:129-36.

${ }^{4}$ Gelzayd EA, Gelfand DW, Rinaldo JA (Jr). Nonspecific duodenitis: a distinct clinical entity? Gastrointest Endosc 1973; 19:131-33.

"Cheli R, Giacosa A. Inflammatory cell count and identification in chronic non-specific duodenitis. Endoscopy 1977; 9:91-5.

"Joffe SN, Lee FD, Blumgart LH. Duodenitis. Clin Gastroenterol 1978; 7:635-50.

'Ferguson A, Sutherland A, MacDonald TT, Allan F. Technique for microdissection and measurement in biopsies of human small intestine. J Clin Pathol 1977; 30:1068-73.

'Zagorulko MP, Puzyrev AA. On proliferation of the epithelium of the duodenal mucosa in ulcerative disease. Arkh Patol 1974; 36:31-5.

'Eastwood GL. Gastrointestinal epithelial renewal. Gastroenterology 1977; 72:962-75.

1"Williamson RCN. Intestinal adaptation. Structural, functional and cytokinetic changes. $N$ Engl J Med 1978; 298:1393-1402. 